\title{
EBSD-Analysis of Microstructural Changes Below Wire-EDMed Surfaces
}

\author{
A. Schwedt ${ }^{1}$, L. Hensgen ${ }^{2}$, J. Dieckmann ${ }^{2}$, A. Klink ${ }^{2}$, F. Klocke ${ }^{2}$, and J. Mayer ${ }^{1}$ \\ 1. Central Facility for Electron Microscopy, RWTH Aachen University, Aachen, Germany. \\ 2. Laboratory for Machine Tools and Production Engineering, RWTH Aachen University, Aachen, \\ Germany.
}

Wire Electro Discharge Machining (W-EDM) is an excellent tool for high-precision machining. If components produced in this way are to be used in mechanically highly demanding applications, an exact knowledge about the microstructure changes below the machined surfaces is indispensible.

An example for such an application are notched monolithic flexure hinges (cf. Fig. 1), which are analyzed within a larger project for the construction of micro-manipulators at RWTH Aachen University. During the bending of such hinges, the rim zone of the notch is undergoing high tensile strain. In order to predict the operating functionality, the microstructural damage introduced by W-EDM cutting therefore needs to be known in dependence of the cutting parameters.

A series of samples from different materials (steels as well as carbide metals) cut under varying conditions by W-EDM has been analysed with EBSD using a Hikari camera with OIM 6.2 by EDAX-TSL, attached to a JEOL JSM-7000F FEG-SEM. Prior to the analyses, cross-sections through the rim zones had to be prepared without introducing additional deformation close to the edges due to the sample preparation. For this purpose ion-polishing using $6 \mathrm{keV} \mathrm{Ar}^{+}$-ions was applied using a Cross-Section Polisher SM-09010 by JEOL.

Depending on the actual parameters of the W-EDM process, several degrees of microstructure alteration were found (cf. Fig. 2). The topmost layer usually is an amorphous or nanocrystalline layer, the crystallites of which would be too small to be measured with EBSD, corresponding to the 'white layers' which can be observed in LOM or BSE images in the SEM. This layer often also implements material from the cutting wire. Below this, a layer of crystalline, but phase-transformed material can be found. The occurrence as well as thickness of these two layers can be significantly reduced by trim cuts following the main cut.

Further below, the base material did not undergo any phase-transformation, but still shows plastic as well as elastic deformation which can be seen in the local misorientations in the base material.

The influence of these alterations on the hinge peformance will be tested.

Acknowledgements:

The authors gratefully acknowledge the financial support by the Deutsche Forschungsgemeinschaft (DFG) within the project "Grundlegende Untersuchungen zu stoffschliissigen Gelenken mit Einsatz in hochgenauen parallelkinematischen Mikromanipulatoren“. 


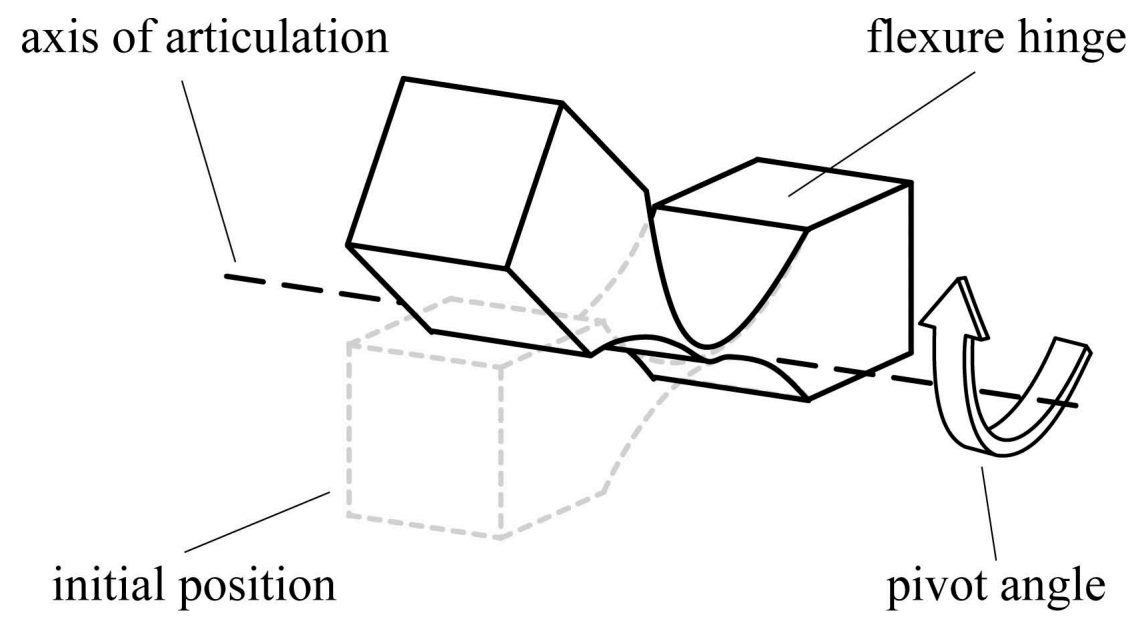

Figure 1. Design of a notched monolithic flexure hinge.

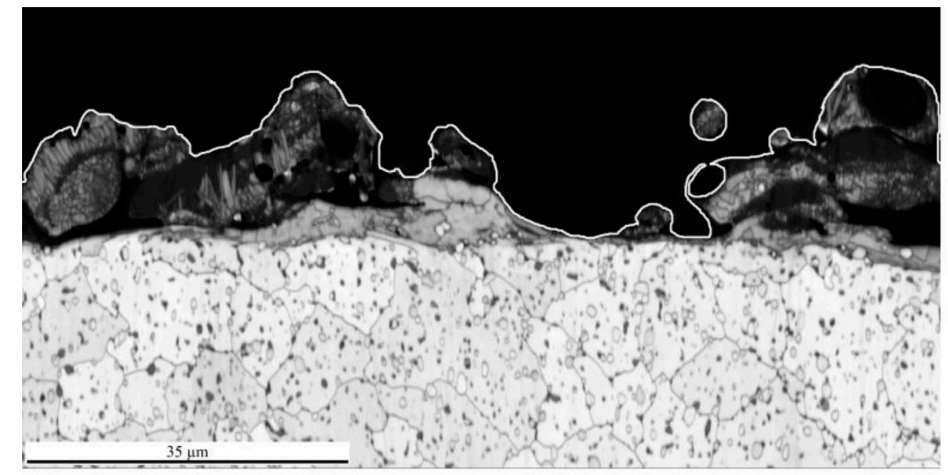

Image Quality Map

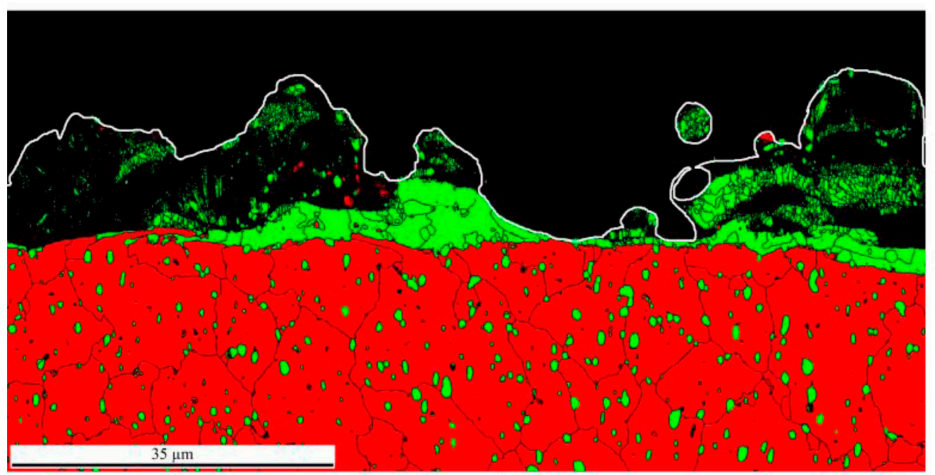

Phase Map $(\mathrm{CI}>0.1)$

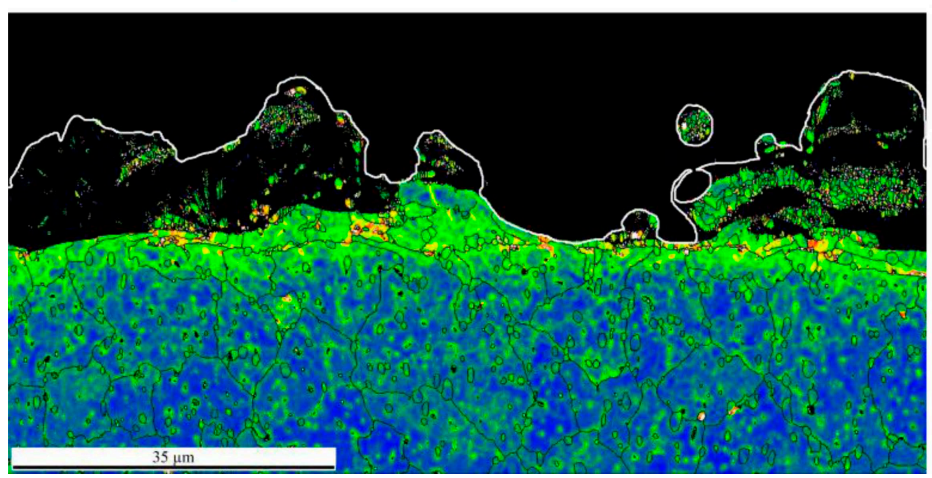

$\operatorname{KAM}\left(300 \mathrm{~nm}, 5^{\circ}\right)$

$0^{\circ} \square 2^{\circ}$

Figure 2. Microstructural changes in terms of crystallinity, phase content and local deformation state after the main W-EDM cut of a X140 CrMoV 5-4-4 PM cold work tool steel. The white line represents the actual surface, marked by Au coating before the preparation. 